\title{
Approach to Chandrasekhar-Kendall-Woltjer State in a Chiral Plasma
}

\author{
Xiao-liang Xia, ${ }^{1}$ Hong Qin, ${ }^{1,2}$ and Qun Wang ${ }^{1}$ \\ ${ }^{1}$ Department of Modern Physics, University of Science and Technology of China, Hefei, Anhui 230026, China \\ ${ }^{2}$ Plasma Physics Laboratory, Princeton University, \\ P.O. Box 451, Princeton, New Jersey 08543, USA
}

\begin{abstract}
We study the time evolution of the magnetic field in a plasma with a chiral magnetic current. The Vector Spherical Harmonic functions (VSH) are used to expand all fields. We define a measure for the Chandrasekhar-Kendall-Woltjer (CKW) state, which has a simple form in VSH expansion. We propose the conditions for a general class of initial momentum spectra that will evolve into the CKW state. For this class of initial conditions, to approach the CKW state, (i) a non-vanishing chiral magnetic conductivity is necessary, and (ii) the time integration of the product of the electric resistivity and chiral magnetic conductivity must grow faster than the time integration of the resistivity. We give a few examples to test these conditions numerically which work very well.
\end{abstract}

\section{INTRODUCTION}

In high energy heavy-ion collisions, two heavy nuclei are accelerated to almost the speed of light and produce very strong electric and magnetic fields at the moment of the collision [1-10]. The magnitude of magnetic fields can be estimated as $e B \sim \gamma v Z e^{2} / R_{A}^{2}$, where $Z$ and $R_{A}$ are the proton number and the radius of the nucleus respectively, $v \approx 1-2 m_{n}^{2} / s$ is the the velocity of the nucleus and $\gamma$ is the Lorentz contraction factor $\gamma \approx \sqrt{s / 2} / m_{n}\left(m_{n}\right.$ is the nucleon mass and $\sqrt{s}$ is the collision energy per nucleon). In $\mathrm{Au}+\mathrm{Au}$ collisions at the Relativistic Heavy Ion Collider (RHIC) with $\sqrt{s}=200 \mathrm{GeV}$, the peak value of the magnetic field at the moment of the collision is about $5 m_{\pi}^{2}\left(m_{\pi}\right.$ is the pion mass) or $1.4 \times 10^{18}$ Gauss. In $\mathrm{Pb}+\mathrm{Pb}$ collisions at the Large Hadron Collider (LHC) with $\sqrt{s}=2.76$ $\mathrm{TeV}$, the peak value of the magnetic field can be 10 times as large as at RHIC. Such high magnetic fields enter strong interaction regime and may have observable effects on the hadronic events. The chiral magnetic effect (CME) is one of them which is the generation of an electric current induced by magnetic fields from of the imbalance of chiral fermions [1, 11, 12]. The CME and other related effects have been widely studied in quark-gluon plasma produced in heavy-ion collisions. The charge separation effect observed in STAR [13, 14] and ALICE [15] experiments are consistent to the CME predictions, although there may be other sources such as collective flows that contribute to the charge separation [16]. The CME has recently been confirmed to exist in materials such as Dirac and Weyl semi-metals [17-19].

In hot and dense matter an imbalance in the number of right-handed quarks and left-handed quarks may be produced through transitions between vacua of different Chern-Simons numbers in some domains of the matter. This is called chiral anomaly and is described by the anomalous conservation law for the axial current,

$$
\partial_{\mu} j_{A}^{\mu}=-\frac{N_{c} e^{2} \sum_{f} Q_{f}^{2}}{8 \pi^{2}} F_{\mu \nu} \tilde{F}^{\mu \nu}-\frac{N_{f} g^{2}}{16 \pi^{2}} F_{\mu \nu}^{a} \tilde{F}_{a}^{\mu \nu},
$$

where $j_{A}^{\mu}=\left(n_{A}, \mathbf{j}_{A}\right)$ denotes the axial 4-vector current with $n_{A}$ being the chiral charge, $N_{c}$ and $N_{f}$ are the number of colors and flavors of quarks respectively, $Q_{f}$ is the electric charge (in the unit of electron charge $e$ ) of the quark with flavor $f, F_{\mu \nu}$ denotes the field strength of the electromagnetic field and $\tilde{F}_{\mu \nu}=\frac{1}{2} \epsilon^{\mu \nu \rho \sigma} F_{\rho \sigma}$ is its dual, $g$ is the strong coupling constant, $F_{\mu \nu}^{a}$ denotes the field strength of the $a$-th gluon with $a=1,2, \cdots, N_{c}^{2}-1$ and $\tilde{F}_{a}^{\mu \nu}=\frac{1}{2} \epsilon^{\mu \nu \rho \sigma} F_{\rho \sigma}^{a}$ is its dual. The first term on the right-hand-side of Eq. (1D) is the anomaly term from electromagnetic fields while the second one is from gluonic fields. In Eq. (1) we have neglected quark masses. For electromagnetic fields we can write $F_{\mu \nu} \tilde{F}^{\mu \nu}$ in the 3 -vector form using $F_{\mu \nu} \tilde{F}^{\mu \nu}=-4 \mathbf{E} \cdot \mathbf{B}$, where $\mathbf{E}$ and $\mathbf{B}$ are the electric and magnetic 3-vector field respectively.

The axial current $j_{A}^{\mu}$ breaks the parity locally and may appear in one event, but it is vanishing when taking event average. With such an imbalance, an electric current can be induced along the magnetic field, so the total electric current can be written as

$$
\mathbf{j}=\sigma \mathbf{E}+\sigma_{\chi} \mathbf{B},
$$

where $\sigma$ and $\sigma_{\chi}$ are the electric and chiral conductivity respectively. Note that $\sigma_{\chi}$ is proportional to the difference between the number of right-handed quarks and left-handed quarks which breaks the 
parity but conserves the time reversal symmetry. This is in contrast with the electric conductivity which breaks the time reversal symmetry but conserves the parity. So the Ohm's current is dissipative (with heat production) while the chiral magnetic current is non-dissipative.

We consider a system of charged fermions in electromagnetic fields. The term of $F_{\mu \nu} \tilde{F}^{\mu \nu}$ or $\mathbf{E} \cdot \mathbf{B}$ in Eq. (1) is actually related to the magnetic helicity $H_{\mathrm{mag}}=\int d^{3} x \mathbf{A} \cdot \mathbf{B}$. Then we can take volume integration of Eq. (11) and obtain

$$
\frac{d}{d t} H_{\text {total }}=0
$$

where the total helicity $H_{\text {total }}$ is defined by combining the magnetic helicity and the chiral charge $N_{A}=\int d^{3} x n_{A}$,

$$
H_{\text {total }}=H_{\text {mag }}-C_{A}^{-1} N_{A}
$$

where $C_{A} \equiv \frac{N_{c} e^{2} \sum_{f} Q_{f}^{2}}{2 \pi^{2}}$. This means that the magnetic helicity and the chiral charge of fermions can be transferred into each other.

The Chandrasekhar-Kendall-Woltjer (CKW) state is a state of the magnetic field which satisfies the following equation

$$
\nabla \times \mathbf{B}=C \mathbf{B}
$$

where $C$ is a constant. The CKW state was first studied by Chandrasekhar, Kendall and Woltjer [20-23] as a force free state. We notice that in a plasma with the chiral magnetic current (2), if the Ohm's current is absent, the system reaches a special CKW state with $C=\sigma_{\chi}$ following the Ampere's law. To our knowledge, this idea was first proposed in [24]. But with the Ohm's current, can the CKW state still be reached? This question can be re-phrased as: what are the conditions under which the CKW state can be reached in a plasma with chiral magnetic currents? In this paper we will answer this question by studying the evolution of magnetic fields with the Maxwell-Chern-Simons equations.

In classical plasma physics, a state satisfying Eq. (5) is called the Taylor state or the Woltjer-Taylor state. It was first found by Woltjer [23] that the CKW state minimizes the magnetic energy for a fixed magnetic helicity. In toroidal plasma devices, such a state is often observed as a self-generated state called reverse field pinch with the distinct feature that the toroidal fields in the center and the edge point to opposite directions. Taylor [25, 26] first argued that the minimization of magnetic energy with a fix magnetic helicity is realized as a selective decay process in a weakly dissipative plasma when the dynamics is dominated by short wavelength structures. Taylor's theory has been questioned and debated intensely, and alternative theories has been proposed [27, 28]. It is certainly interesting that both classical plasmas and chiral plasmas have the tendency to evolve towards such a state, which suggests that the two systems may share certain dynamics features responsible for the emerging of the state. A brief discussion in this aspect is given in the paper as well.

The paper is organized as follows. In Section II] we start with the Maxwell-Chern-Simons equations and define a global measure for the CKW state by the magnetic field and the electric current. In Section III], we expand all fields in Vector Spherical Harmonic (VSH) functions. The inner products have simple forms in the VSH expansion. The parity of a quantity can be easily identified in the VSH form. We give in Section IV the solution to the Maxwell-Chern-Simons equations for each mode in the VSH expansion. The conditions for the CKW state are given in Section V] In Section VI we test these conditions by examples including the ones with constant and self-consistently determined $\sigma_{\chi}$. We also generalize the momentum spectra at the initial time from a power to a polynomial pre-factor of scalar momentum in Section VII. The summary and conclusions are made in the last section.

\section{MAXWELL-CHERN-SIMONS EQUATIONS AND CKW STATE}

We start from Maxwell-Chern-Simons equations or anomalous Maxwell equations,

$$
\begin{aligned}
\nabla \times \mathbf{B} & =\sigma \mathbf{E}+\sigma_{\chi} \mathbf{B} \\
\nabla \times \mathbf{E} & =-\frac{\partial}{\partial t} \mathbf{B}, \\
\nabla \cdot \mathbf{B} & =0 \\
\nabla \cdot \mathbf{E} & =0
\end{aligned}
$$


where we have included the induced current $\mathbf{j}=\sigma \mathbf{E}+\sigma_{\chi} \mathbf{B}$ and neglected the displacement current $\frac{\partial}{\partial t} \mathbf{E}$. We have also dropped the external charge and current density. We assume that $\sigma$ and $\sigma_{\chi}$ depend on $t$ only.

Taking curl of Eq. (6) and using Eqs. (78), we obtain

$$
\sigma \frac{\partial}{\partial t} \mathbf{B}=\nabla^{2} \mathbf{B}+\sigma_{\chi} \nabla \times \mathbf{B}
$$

A similar equation for $\mathbf{E}$ can also be derived but we will not consider it in the current study. To measure whether the CKW state is reached in the evolution of the magnetic field, we introduce the quantity

$$
\cos ^{2} \theta(t) \equiv \frac{\left[\int d^{3} x(\nabla \times \mathbf{B}) \cdot \mathbf{B}\right]^{2}}{\int d^{3} x(\nabla \times \mathbf{B}) \cdot(\nabla \times \mathbf{B}) \int d^{3} x \mathbf{B} \cdot \mathbf{B}}=\frac{\langle\mathbf{j}, \mathbf{B}\rangle^{2}}{\langle\mathbf{j}, \mathbf{j}\rangle\langle\mathbf{B}, \mathbf{B}\rangle},
$$

where we have used the notation for the inner product $\langle\mathbf{X}, \mathbf{Y}\rangle \equiv \int d^{3} x(\mathbf{X} \cdot \mathbf{Y})$ for any vector field $\mathbf{X}$ and $\mathbf{Y}$. According to the Cauchy-Schwartz inequality

$$
\langle\mathbf{j}, \mathbf{j}\rangle\langle\mathbf{B}, \mathbf{B}\rangle \geq\langle\mathbf{j}, \mathbf{B}\rangle^{2},
$$

we have $\cos ^{2} \theta(t) \leq 1$, where the equality holds only in the case of $\mathbf{j} \|( \pm \mathbf{B})$ when the CKW state is reached [28]. We assume that $\cos ^{2} \theta(t)$ is a smooth function of $t$. The condition that the CKW state is reached can be given by

$$
\lim _{t \rightarrow \infty} \cos ^{2} \theta(t)=1 .
$$

Note that $\cos ^{2} \theta(t)$ should not exactly be equal to 1 , since $\cos ^{2} \theta(t)$ is a smooth function of $t$ and bounded by the upper limit 1 .

To see the time evolution of $\cos ^{2} \theta(t)$, it is helpful to write inner products in simple forms, which we will do in the next section.

\section{EXPANSION IN VECTOR SPHERICAL HARMONIC FUNCTIONS}

In this section, we will expand all fields in the basis of Vector Spherical Harmonic function (VSH), with which we can put inner products into a simple and symmetric form.

\section{A. Expansion in VSH}

The quantities we use to express $\cos ^{2} \theta(t)$ in Eq. (11) are $\mathbf{B}=\nabla \times \mathbf{A}$ and $\mathbf{j}=\nabla \times \mathbf{B}=\nabla \times(\nabla \times \mathbf{A})$. We can extend the series to include more curls,

$$
\mathbf{A}, \mathbf{B}=\nabla \times \mathbf{A}, \mathbf{j}=(\nabla \times)^{2} \mathbf{A}, \cdots,(\nabla \times)^{n} \mathbf{A}, \cdots,
$$

So the inner products can be written as $\left\langle(\nabla \times)^{n_{1}} \mathbf{A},(\nabla \times)^{n_{2}} \mathbf{A}\right\rangle$ with $n_{1}$ and $n_{2}$ are non-negative integers. To find an unified form for the fields in this series, we can expand $\mathbf{A}(\mathbf{x}, t)$ in the Coulomb gauge in $\mathrm{VSH}$

$$
\mathbf{A}(\mathbf{x}, t)=\frac{1}{\pi} \sum_{l, m} \int_{0}^{\infty} d k k\left[\alpha_{l m}^{+}(t, k) \mathbf{W}_{l m}^{+}(\mathbf{x} ; k)-\alpha_{l m}^{-}(t, k) \mathbf{W}_{l m}^{-}(\mathbf{x} ; k)\right],
$$

where $k=|\mathbf{k}|$ is the scalar momentum and $l, m$ are the quantum number of the angular momentum and the angular momentum along a particular direction respectively. $\mathbf{W}_{l m}^{ \pm}(\mathbf{x} ; k)$ are divergence-free vector fields which can be expressed in term of VSH $\mathbf{X}_{l m}=\frac{-i}{\sqrt{l(l+1)}} \mathbf{r} \times \nabla Y_{l m}$ [29]. The explicit form of $\mathbf{W}_{l m}^{ \pm}$can be found in, e.g., Ref. [30, 31]. The orthogonal basis functions $\mathbf{W}_{l m}^{ \pm}(\mathbf{x} ; k)$ satisfy the following orthogonality relations,

$$
\int d^{3} x \mathbf{W}_{l_{1} m_{1}}^{s_{1} *}(\mathbf{x} ; k) \cdot \mathbf{W}_{l_{2} m_{2}}^{s_{2}}\left(\mathbf{x} ; k^{\prime}\right)=\frac{\pi}{k^{2}} \delta\left(k-k^{\prime}\right) \delta_{l_{1} l_{2}} \delta_{m_{1} m_{2}} \delta_{s_{1} s_{2}},
$$


where $s_{1}, s_{2}= \pm$. Note that $\mathbf{W}_{l m}^{ \pm}(\mathbf{x} ; k)$ themselves are CKW states satisfying

$$
\nabla \times \mathbf{W}_{l m}^{ \pm}(\mathbf{x} ; k)= \pm k \mathbf{W}_{l m}^{ \pm}(\mathbf{x} ; k),
$$

and are divergence-free, $\nabla \cdot \mathbf{W}_{l m}^{ \pm}=0$, so we can expand any divergence-free vector fields in $\mathbf{W}_{l m}^{ \pm}$. We note that $\mathbf{A}(\mathbf{x}, t)$ is real while $\alpha_{l m}^{ \pm}(t, k)$ and $\mathbf{W}_{l m}^{ \pm}(\mathbf{x} ; k)$ are complex.

Taking curls of Eq. (15) and using Eq. (17), we can expand all quantities in the series (14) in VSH,

$$
(\nabla \times)^{n} \mathbf{A}=\frac{1}{\pi} \sum_{l, m} \int_{0}^{\infty} d k k^{n+1}\left[\alpha_{l m}^{+}(t, k) \mathbf{W}_{l m}^{+}(\mathbf{x} ; k)+(-1)^{n+1} \alpha_{l m}^{-}(t, k) \mathbf{W}_{l m}^{-}(\mathbf{x} ; k)\right] .
$$

In particular the expansions of $\mathbf{B}$ and $\mathbf{j}$ are

$$
\begin{aligned}
\mathbf{B}(\mathbf{x}, t) & =\frac{1}{\pi} \sum_{l, m} \int_{0}^{\infty} d k k^{2}\left[\alpha_{l m}^{+}(t, k) \mathbf{W}_{l m}^{+}(\mathbf{x} ; k)+\alpha_{l m}^{-}(t, k) \mathbf{W}_{l m}^{-}(\mathbf{x} ; k)\right], \\
\mathbf{j}(\mathbf{x}, t) & =\frac{1}{\pi} \sum_{l, m} \int_{0}^{\infty} d k k^{3}\left[\alpha_{l m}^{+}(t, k) \mathbf{W}_{l m}^{+}(\mathbf{x} ; k)-\alpha_{l m}^{-}(t, k) \mathbf{W}_{l m}^{-}(\mathbf{x} ; k)\right] .
\end{aligned}
$$

Note that the signs in front of $\alpha_{l m}^{-}$are $(-1)^{n+1}$, which are related to the parity of the quantity.

\section{B. Inner products}

We can put the general inner products into a simple form by using the orthogonality relation (16),

$$
\left\langle(\nabla \times)^{n_{1}} \mathbf{A},(\nabla \times)^{n_{2}} \mathbf{A}\right\rangle=\int_{0}^{\infty} d k k^{n_{1}+n_{2}}\left[g_{+}(t, k)+(-1)^{n_{1}+n_{2}} g_{-}(t, k)\right],
$$

where $g_{ \pm}(t, k)$ are defined by

$$
g_{ \pm}(t, k)=\frac{1}{\pi} \sum_{l, m}\left|\alpha_{l m}^{ \pm}(t, k)\right|^{2}
$$

We note that $g_{ \pm}(t, k)$ are positive definite. In deriving Eq. (20) we have used the fact that $\mathbf{A}$ is real so that $\left\langle(\nabla \times)^{n_{1}} \mathbf{A},(\nabla \times)^{n_{2}} \mathbf{A}\right\rangle=\left\langle(\nabla \times)^{n_{1}} \mathbf{A}^{*},(\nabla \times)^{n_{2}} \mathbf{A}\right\rangle$. For convenience, we use the following short-hand notation for the integral

$$
\int k^{n} \equiv \int_{0}^{\infty} d k k^{n}\left[g_{+}(t, k)+(-1)^{n} g_{-}(t, k)\right]
$$

From Eq. (201) it is easy to verify

$$
\left\langle(\nabla \times)^{n_{1}} \mathbf{A},(\nabla \times)^{n_{2}} \mathbf{A}\right\rangle=\left\langle(\nabla \times)^{n_{3}} \mathbf{A},(\nabla \times)^{n_{4}} \mathbf{A}\right\rangle
$$

for $n_{1}+n_{2}=n_{3}+n_{4}$. This is consistent to the identity $\langle\nabla \times \mathbf{X}, \mathbf{Y}\rangle=\langle\mathbf{X}, \nabla \times \mathbf{Y}\rangle$. In Table (II), we list the VSH forms of some inner products that we are going to study later in this paper.

\begin{tabular}{|c|c|c|c|}
\hline Quantity & VSH form & Short-hand & Parity \\
\hline$\langle\mathbf{j}, \mathbf{j}\rangle$ & $\int_{0}^{\infty} d k k^{4}\left[g_{+}(t, k)+g_{-}(t, k)\right]$ & $\int k^{4}$ & even \\
\hline$\langle\mathbf{j}, \mathbf{B}\rangle$ & $\int_{0}^{\infty} d k k^{3}\left[g_{+}(t, k)-g_{-}(t, k)\right]$ & $\int k^{3}$ & odd \\
\hline$\langle\mathbf{B}, \mathbf{B}\rangle$ & $\int_{0}^{\infty} d k k^{2}\left[g_{+}(t, k)+g_{-}(t, k)\right]$ & $\int k^{2}$ & even \\
\hline$\langle\mathbf{A}, \mathbf{B}\rangle$ & $\int_{0}^{\infty} d k k\left[g_{+}(t, k)-g_{-}(t, k)\right]$ & $\int k^{1}$ & odd \\
\hline
\end{tabular}

TABLE I: Some inner products in VSH expansion. 


\section{Parity and helicity}

From Eq. (17), the parity transformation $\mathbf{x} \rightarrow-\mathbf{x}$ is equivalent to the interchange of the + and mode. In the series (14), the quantity $(\nabla \times)^{n} \mathbf{A}$ is parity-even/parity-odd (P-even/P-odd) for odd/even $n$. For instance, $\mathbf{A}, \mathbf{B}$ and $\mathbf{j}$ are P-odd, P-even and P-odd respectively.

Also the inner product $\int k^{n}$ is $\mathrm{P}$-even/P-odd for even/odd $n$, see the last column of Table (II) where the magnetic helicity $H=\langle\mathbf{A}, \mathbf{B}\rangle=\int k$ is $\mathrm{P}$-odd, and the magnetic energy $W=\frac{1}{2}\langle\mathbf{B}, \mathbf{B}\rangle=\frac{1}{2} \int k^{2}$ is P-even.

For a momentum spectrum containing only $g_{+}$, the helicity is positive. If such a magnetic field can approach the CKW state, it means $\mathbf{j} \| \mathbf{B}$ because $\langle\mathbf{j}, \mathbf{B}\rangle$ is also positive for $g_{+}$mode. In contrast it would mean $\mathbf{j} \|-\mathbf{B}$ for a momentum spectrum containing only $g_{-}$.

\section{SOLVING MAXWELL-CHERN-SIMONS EQUATIONS IN VSH}

The evolution equation of $\mathbf{B}$ in (10) can be transformed into equations for $\alpha_{l m}^{ \pm}(t, k)$ or equivalently $g_{ \pm}(t, k)$ with Eqs. (1916),

$$
\begin{aligned}
\frac{d}{d t} \alpha_{l m}^{ \pm}(t, k) & =\eta\left(-k^{2} \pm \sigma_{\chi} k\right) \alpha_{l m}^{ \pm}(t, k), \\
\frac{d}{d t} g_{ \pm}(t, k) & =2 \eta\left(-k^{2} \pm \sigma_{\chi} k\right) g_{ \pm}(t, k),
\end{aligned}
$$

where $\eta=\frac{1}{\sigma}$ is the electric resistivity.

The solution of $g_{ \pm}(t, k)$ is in the form

$$
g_{ \pm}(t, k)=g_{ \pm}\left(t_{0}, k\right) e^{-2 k^{2} \Lambda(t) \pm 2 k \Theta(t)}
$$

where $g_{ \pm}\left(t_{0}, k\right)$ denote the values at the initial time $t_{0}$, and $\Lambda(t)$ and $\Theta(t)$ are defined by

$$
\Lambda(t)=\int_{t_{0}}^{t} \eta(\tau) d \tau, \quad \Theta(t)=\int_{t_{0}}^{t} \eta(\tau) \sigma_{\chi}(\tau) d \tau
$$

Note that both $\Lambda(t)$ and $\Theta(t)$ are positive.

Alternatively we can rescale time by using $\Lambda$ as a new evolution parameter, and rewrite $\Theta(\Lambda)=$ $\int_{0}^{\Lambda} \sigma_{\chi}(\Lambda) d \Lambda$, i.e., $\Theta(\Lambda)$ is the integrated value of $\sigma_{\chi}(t)$ from 0 to $\Lambda$.

There is a competition between $\sigma$ and $\sigma_{\chi}$ for approaching or departing the CKW state. Large values of $\Theta(t)$ is favored for the CKW state. We will show that it is indeed determined by the increasing ratio of $\Lambda(t)$ to $\Theta(t)$.

Note that in Eq. (25) changing $\sigma_{\chi} \rightarrow-\sigma_{\chi}$ is equivalent to interchanging the positive and negative modes, therefore we can assume $\sigma_{\chi}>0$ in this paper without loss of generality.

\section{CONDITIONS FOR CKW STATE}

In this section we will study the evolution of the fields in the basis of VSH and look for the conditions for the CKW state.

From Eq. (11) we obtain $\cos ^{2} \theta(t)$ in VSH,

$$
\cos ^{2} \theta(t)=\frac{\left\{\int d k k^{3}\left[g_{+}(t, k)-g_{-}(t, k)\right]\right\}^{2}}{\int d k k^{4}\left[g_{+}(t, k)+g_{-}(t, k)\right] \times \int d k k^{2}\left[g_{+}(t, k)+g_{-}(t, k)\right]} .
$$

To verify $\cos ^{2} \theta(t)<1$, it is better to rewrite $\cos ^{2} \theta(t)$ in a more symmetric form,

$$
\cos ^{2} \theta(t)=\frac{\int_{0}^{\infty} d k_{1} d k_{2}\left(2 k_{1}^{3} k_{2}^{3}\right) \times\left[g_{+}\left(t, k_{1}\right)-g_{-}\left(t, k_{1}\right)\right]\left[g_{+}\left(t, k_{2}\right)-g_{-}\left(t, k_{2}\right)\right]}{\int_{0}^{\infty} d k_{1} d k_{2}\left(k_{1}^{4} k_{2}^{2}+k_{1}^{2} k_{2}^{4}\right) \times\left[g_{+}\left(t, k_{1}\right)+g_{-}\left(t, k_{1}\right)\right]\left[g_{+}\left(t, k_{2}\right)+g_{-}\left(t, k_{2}\right)\right]} .
$$

The difference between the denominator and numerator is

$$
\text { Diff } \geq 8 \int_{0}^{\infty} d k_{1} d k_{2} k_{1}^{3} k_{2}^{3} g_{+}\left(t, k_{1}\right) g_{-}\left(t, k_{2}\right) \geq 0,
$$


where we have used $k_{1}^{4} k_{2}^{2}+k_{1}^{2} k_{2}^{4} \geq 2 k_{1}^{3} k_{2}^{3}$ in the first inequality. From the inequality (29) it is obvious that $\cos ^{2} \theta(t) \leq 1$, where the equality holds for $k_{1}=k_{2}$ and one of $g_{+}(t, k)$ and $g_{-}(t, k)$ is zero.

Therefore we have two conditions under which $\lim _{t \rightarrow \infty} \cos ^{2} \theta(t)=1$ is satisfied:

$$
\begin{aligned}
& (1) g_{-}(t, k) \rightarrow 0 \text {, leaving only } g_{+}(t, k), \\
& (2) g_{+}(t, k) \rightarrow \delta\left(k-k_{\mathrm{c}}\right),
\end{aligned}
$$

where $k_{\mathrm{c}}(t)$ is the central momentum of $g_{+}(t, k)$ during evolution. Both conditions can be physically understood. The first condition is actually the presence of $\sigma_{\chi}$ (we have assumed $\sigma_{\chi}>0$ ), which makes positive modes grow with time while negative modes decay away. It means that the CKW state should contain only positive (or negative) helicity mode only, which is reasonable because the CKW state is the eigenstate of the curl operator. For the second condition, we notice that $\mathbf{W}_{l m}^{ \pm}$bases themselves are CKW states from Eq. (17), therefore one single mode in the expansion (19) is natually the CKW state. The authors of Ref. [30] observed $g_{+}(t, k) \rightarrow \delta\left(k-k_{\mathrm{c}}\right)$ in the evolution to the CKW state. However, the delta function is not well defined mathematically, so the second condition is hard to implement and we must find a better one to replace it.

With the solutions for $g_{ \pm}(t, k)$ in Eq. (25), we obtain the time behavior of $\cos ^{2} \theta(t)$ from Eq. (27)

$$
\cos ^{2} \theta(t)=\frac{\left[I_{3}^{+}(t)-I_{3}^{-}(t)\right]^{2}}{\left[I_{4}^{+}(t)+I_{4}^{-}(t)\right]\left[I_{2}^{+}(t)+I_{2}^{-}(t)\right]},
$$

where the time functions $I_{n}^{ \pm}(t)$ are defined by

$$
I_{n}^{ \pm}(t)=\int_{0}^{\infty} d k k^{n} g_{ \pm}\left(t_{0}, k\right) e^{-2 k^{2} \Lambda(t) \pm 2 k \Theta(t)} .
$$

Here $n=2,3,4$ are the powers of $k$ in the integrals for $\langle\mathbf{B}, \mathbf{B}\rangle,\langle\mathbf{j}, \mathbf{B}\rangle$ and $\langle\mathbf{j}, \mathbf{j}\rangle$, respectively.

If the initial spectrum functions $g_{ \pm}\left(t_{0}, k\right)$ contain only $k^{r}(r$ is a real number $), e^{-2 C_{1} k}$ or $e^{-C_{2}^{2} k^{2}}$ $\left(C_{1}\right.$ and $C_{2}$ are real constants), the integrals $I_{n}^{ \pm}$are just Gaussian-like integrals and easy to deal with. In this paper we assume that $g_{ \pm}\left(t_{0}, k\right)$ take the following form

$$
g_{ \pm}\left(t_{0}, k\right) \sim k^{r} e^{-2 C_{1} k-C_{2}^{2} k^{2}} .
$$

A typical example of magnetic fields expressed in such a form is the Hopf state [32]. Although this assumption narrows the scope of $g_{ \pm}\left(t_{0}, k\right)$, it is still general enough: these three kinds of functions are widely used in other fields of physics. It is natural to combine $g_{ \pm}\left(t_{0}, k\right)$ with $k^{n} e^{-2 k^{2} \Lambda(t) \pm 2 k \Theta(t)}$ in the integrand of $I_{n}^{ \pm}(t)$ and re-define the time function $I_{r+n}^{ \pm}(t)$ as

$$
I_{r+n}^{ \pm}(t) \equiv \int_{0}^{\infty} d k k^{r+n} \exp \left\{-\left[2 \Lambda(t)+C_{2}^{2}\right] k^{2}+2\left[-C_{1} \pm \Theta(t)\right] k\right\}
$$

where $r$ is the power of $k$ in the initial spectrum functions $g_{ \pm}\left(t_{0}, k\right)$. Note that $I_{-1}^{ \pm}$does not converge, so we assume $r+n>-1$. By changing the integral variable $k \rightarrow \kappa=k \sqrt{2 \Lambda(t)+C_{2}^{2}}$ where $2 \Lambda(t)+C_{2}^{2}$ is always positive by definition, we can rewrite $I_{r+n}^{ \pm}(t)$ in the form

$$
I_{r+n}^{ \pm}(t)=\frac{G_{r+n}\left(a^{ \pm}\right)}{\left[2 \Lambda(t)+C_{2}^{2}\right]^{(r+n+1) / 2}},
$$

where $G_{r+n}\left(a^{ \pm}\right)$are defined by

$$
G_{r+n}\left(a^{ \pm}\right) \equiv \int_{0}^{\infty} d \kappa \kappa^{r+n} e^{-\kappa^{2}+2 a^{ \pm} \kappa},
$$

with the time functions $a^{ \pm}(t)$ by

$$
a^{ \pm}(t)=\frac{ \pm \Theta(t)-C_{1}}{\sqrt{2 \Lambda(t)+C_{2}^{2}}} .
$$

We rewrite $\cos ^{2} \theta(t)$ in Eq. (31) as a function of $a^{ \pm}(t)$ through $G_{r+n}\left(a^{ \pm}\right)$,

$$
\cos ^{2} \theta(t)=\frac{\left[G_{r+3}\left(a^{+}\right)-G_{r+3}\left(a^{-}\right)\right]^{2}}{\left[G_{r+4}\left(a^{+}\right)+G_{r+4}\left(a^{-}\right)\right]\left[G_{r+2}\left(a^{+}\right)+G_{r+2}\left(a^{-}\right)\right]} .
$$


We give relevant properties of $G_{r+n}(a)$ in Appendix A One property is that $G_{r+n}(a)$ are monotonically increasing functions of $a$, which approach zero at $a \rightarrow-\infty$, but rise sharply to $+\infty$ at $a \rightarrow+\infty$. By Eq. (37), if $\Theta(t)$ grows faster than $\sqrt{\Lambda(t)}$ with $t$, we have $a^{ \pm}(t) \rightarrow \pm \infty$ as $t \rightarrow+\infty$. As time goes on, $G_{r+n}\left(a^{+}\right)$associated with positive modes will grow up but $G_{r+n}\left(a^{-}\right)$associated with negative modes will decay away. At $t \rightarrow+\infty$, Eq. (38) becomes

$$
\cos ^{2} \theta(t) \approx \frac{\left[G_{r+3}\left(a^{+}\right)\right]^{2}}{G_{r+4}\left(a^{+}\right) G_{r+2}\left(a^{+}\right)} .
$$

This fulfills the first condition for the CKW state in (30), i.e. only the positive modes survive at the end of the time evolution.

We also show in Appendix A that the right hand side of Eq. (39) tend to 1 if and only if $a^{+} \rightarrow+\infty$. To make $\cos ^{2} \theta(t) \rightarrow 1$ at $t \rightarrow+\infty$ requires $a^{+}(t) \rightarrow+\infty$, or

$$
\lim _{t \rightarrow \infty} \frac{\Theta(t)-C_{1}}{\sqrt{2 \Lambda(t)+C_{2}^{2}}} \rightarrow+\infty .
$$

So we can summarize the conditions for the CKW state to be reached in time evolution:

$$
\begin{aligned}
& \text { (1) } \sigma_{\chi} \neq 0, \\
& \text { (2) } \lim _{t \rightarrow \infty} \frac{\Theta(t)}{\sqrt{\Lambda(t)}} \rightarrow+\infty .
\end{aligned}
$$

Note that $\sigma_{\chi}$ or $\Theta(t)$ plays an essential role: it makes negative modes more and more suppressed while making positive modes blow up as time goes on. At the same time it makes $a^{+} \rightarrow+\infty$ at $t \rightarrow+\infty$ so that $\cos ^{2} \theta(t) \rightarrow 1$.

In heavy-ion collisions, $\sigma$ and $\sigma_{\chi}$ are decreasing functions of $t$ as the result of the expansion of the QGP matter. It is natural to assume that $\sigma$ and $\sigma_{\chi}$ fall with time in power laws [33], $\sigma(t) \sim t^{-\alpha}$ and $\sigma_{\chi}(t) \sim t^{-\beta}$, where $\alpha, \beta>0$. This can be justified by the fact that $\sigma \sim 5.8 \frac{T}{T_{c}} \mathrm{MeV}[34]$ and $\sigma_{\chi} \sim \mu_{5}$ [35], where both the temperature $T$ and the chiral chemical potential $\mu_{5}$ decrease with time in power laws in expansion. In this case we have $\Lambda(t) \sim t^{1+\alpha}, \Theta(t) \sim t^{1+\alpha-\beta}$ following Eq. (26), and $a^{+} \sim t^{(1+\alpha) / 2-\beta}$, the condition for the CKW state now becomes

$$
\sigma_{\chi} \neq 0, \text { and } \beta<\frac{1+\alpha}{2} \text {. }
$$

The above condition is very easy to check and it is one of the most useful and practical criteria in this paper.

The fact that a large $\sigma_{\chi}$ will bring the system to the CKW state shows that a non-Ohmic current may play a crucial role in the process of reaching the CKW state. This suggests that in classical plasmas systems, a non-Ohmic current, e.g., the Hall current, could produce the same effect. In a classical system with the Hall current and negligible flow velocity, the evolution of the magnetic field is governed by

$$
\sigma \frac{\partial \mathbf{B}}{\partial t}=\nabla^{2} \mathbf{B}-\frac{\sigma}{n e} \nabla \times(\mathbf{j} \times \mathbf{B})
$$

where $n$ is the density of the plasma, $e$ is electron charge, and the last is the Hall current term. Obviously, with a small resistivity, the system approaches equilibrium when the CKW state is reached.

\section{EXAMPLES AND TESTS OF CONDITIONS}

In this section we will look at examples of the CKW state to test the conditions we propose in the last section.

\section{A. With $g_{+}$only}

As the first example, let us consider an initial spectrum with only $g_{+}\left(t_{0}, k\right)$ without $g_{-}\left(t_{0}, k\right)$. We assume $g_{+}\left(t_{0}, k\right)$ has the following form,

$$
g_{+}\left(t_{0}, k\right)=4 H_{0} L^{3} k e^{-2 L k},
$$


where $L$ characterizes the length scale of the magnetic field, $H_{0}$ is the initial magnetic helicity. The normalization constant is chosen to be $4 H_{0} L^{3}$ which gives the initial magnetic helicity of the spectrum,

$$
H_{0}=\int_{0}^{\infty} d k k g_{+}\left(t_{0}, k\right) \text {. }
$$

From Eq. (39) we obtain

$$
\cos ^{2} \theta(t)=\frac{\left[G_{4}(a)\right]^{2}}{G_{5}(a) G_{3}(a)}
$$

where

$$
a(t)=\frac{\Theta(t)-L}{\sqrt{2 \Lambda(t)}}
$$

is given by Eq. (37) with $C_{1}=L$ and $C_{2}=0$. Here we have suppressed the superscript of $a^{+}$and simply denote $a \equiv a^{+}$.

To verify our conditions for the CKW state, we consider following cases:

$$
\begin{aligned}
& \text { a) } \sigma=\sigma_{0}, \sigma_{\chi}=\sigma_{\chi 0} \\
& \text { b) } \sigma=\sigma_{0}, \sigma_{\chi}=0 \\
& \text { c) } \sigma=\sigma_{0}, \sigma_{\chi}=\sigma_{\chi 0}\left(t_{0} / t\right)^{1 / 2} \\
& \text { d) } \sigma=\sigma_{0}\left(t_{0} / t\right)^{1 / 3}, \sigma_{\chi}=\sigma_{\chi 0}\left(t_{0} / t\right)^{1 / 2}
\end{aligned}
$$

In case a) both $\sigma$ and $\sigma_{\chi}$ are constants, which is used in Refs. [9, 10] to calculate the magnetic field in medium. In this case, we have $\alpha=\beta=0$ and $a(t) \sim t^{1 / 2}$ in late time which satisfies the condition $\beta<\frac{1+\alpha}{2}$, and we can see the effect of non-vanishing constant $\sigma_{\chi}$. Such an effect can be seen by comparing with case b) in which we switch off $\sigma_{\chi}$. In case c) $\sigma$ is still a constant as same as case a), but $\sigma_{\chi} \sim t^{-1 / 2}$ is chosen to break the condition $\beta<\frac{1+\alpha}{2}$ with $\alpha=0$ and $\beta=1 / 2$. In case d), the values $\alpha=1 / 3$ and $\beta=1 / 2$ are used in Refs. 33,36 , which are thought to be more reasonable in heavy-ion collisions. But we note that in real situations of heavy-ion collisions, the time behaviors of $\sigma$ and $\sigma_{\chi}$ can be very complicated (may not follow power laws), but our conditions in (41) are still applicable.
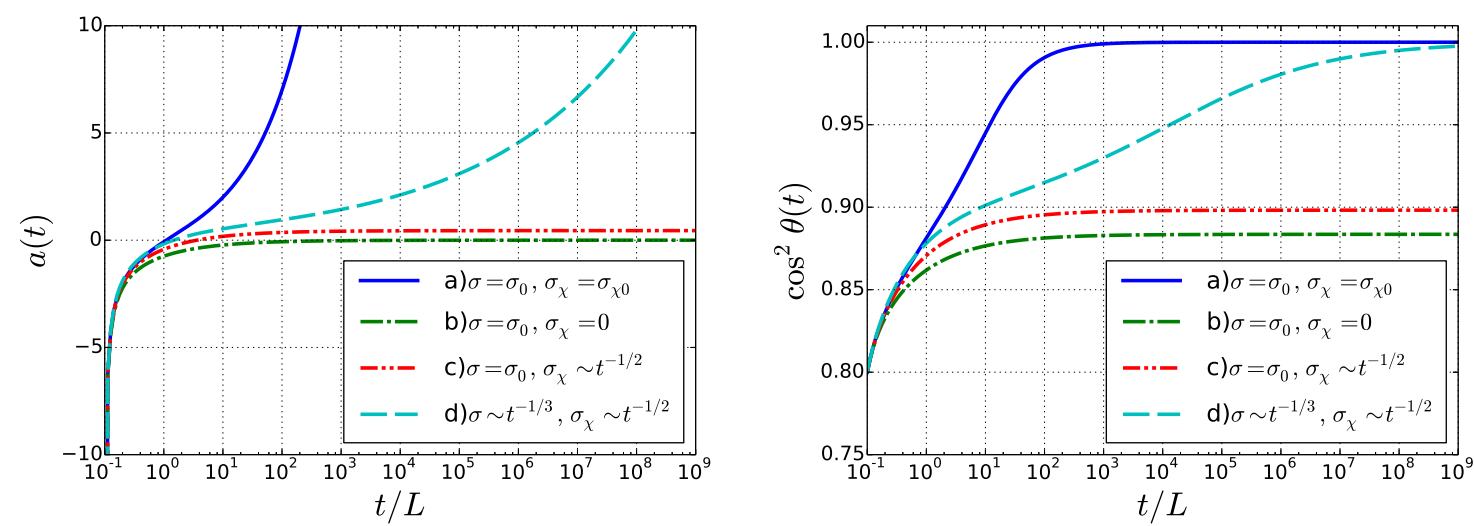

FIG. 1: (color online) The time functions of $a(t)$ (left panel) and $\cos ^{2} \theta(t)$ (right panel) in different cases. In case a) (blue solid) and d) (cyan dashed), $a(t)$ increase toward $+\infty$ and $\cos ^{2} \theta(t)$ tend toward 1 . In case b) (green dot-dashed) and c) (red dot-dot-dashed), $a(t)$ tend toward 0 and $\frac{\sigma_{\chi 0}}{\sigma_{0}} \sqrt{2 t_{0} \sigma_{0}}$ respectively, and $\cos ^{2} \theta(t)$ tend toward saturation values less than 1.

For numerical simulation, we choose $\sigma_{0}=\sigma_{\chi 0}=1 / L$ and $t_{0}=0.1 L$. The results are shown in Fig. 1. Indeed in case a) and d), the CKW state can be reached. As $t$ goes from $t_{0}$ to $\infty$, according to Eqs. (46. 47. A7), $a(t)$ evolves from $-\infty$ to $\infty$, and $\cos ^{2} \theta(t)$ evolves from 0.8 to 1 . In case b) and c) the condition (42) is not satisfied, the CKW state is inaccessible. Indeed the simulation shows that it is true since $a(t)$ tends toward 0 and $\frac{\sigma_{\chi 0}}{\sigma_{0}} \sqrt{2 t_{0} \sigma_{0}}$ in case b) and c) at $t \rightarrow \infty$, respectively. Even though 
$a(t)$ and $\cos ^{2} \theta(t)$ increase with $t$, we have $\cos ^{2} \theta(t) \rightarrow 0.884$ and 0.898 at $t \rightarrow+\infty$ corresponding to $a \rightarrow 0$ and $a \rightarrow \frac{\sigma_{\chi 0}}{\sigma_{0}} \sqrt{2 t_{0} \sigma_{0}}$ respectively. All these results show that the conditions work well.

But we should point out that constant $\sigma$ and $\sigma_{\chi}$ or even the power law decayed $\sigma$ and $\sigma_{\chi}$ may not be physical since once $\sigma_{\chi}$ persists for a long time, $\Theta(t)$ growing faster than $\sqrt{\Lambda(t)}$ will make some physical quantities diverge. We look at the magnetic helicity $H$ and the magnetic energy $W$,

$$
\begin{gathered}
H=\int k=4 H_{0} L^{3} \frac{G_{2}(a)}{[2 \Lambda(t)]^{3 / 2}}, \\
W=\frac{1}{2} \int k^{2}=2 H_{0} L^{3} \frac{G_{3}(a)}{[2 \Lambda(t)]^{2}} .
\end{gathered}
$$

The numerical results of the magnetic helicity are shown in Fig. 2. The results of the magnetic energy are similar. In case b) and c), since $a(t)$ and $G_{n}(a)$ converge to constants, but $\Lambda(t)$ keeps growing, both $H$ and $W$ finally decay to zero following Eq. (49). However in case a) and d), $a(t)$ and $G_{n}(a)$ increase to $+\infty$ in late time, and from $\mathrm{Eq}$ (A6) $G_{n}(a) \sim a^{n} \exp \left(a^{2}\right)$ grow much faster than $[\Lambda(t)]^{(n+1) / 2}$ to make $H$ and $W$ blow up.

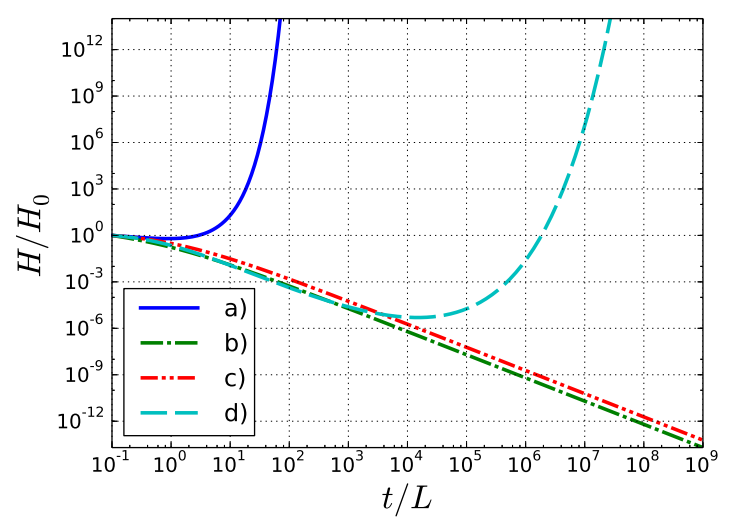

FIG. 2: (color online) The time dependence of the magnetic helicity $H$ in different cases. In case a) (blue solid) and d) (cyan dashed), $H$ blow up at some time. In case b) (green dot-dashed) and c) (red dot-dot-dashed), $H$ decay to zero in late time.

From Eq. (24), we see that the spectrum $g_{+}(t, k)$ grows exponentially in time for $k<\sigma_{\chi}$, such an instability has been discussed in [9, 37], see also [38]. This instability is the source of the divergence of $H$ and $W$. Such an unphysical inflation can be understood: the appearance of $\sigma_{\chi} \mathbf{B}$ in the induced current leads to the positive feedback that the magnetic field itself induces the magnetic field. If we put no constraint on $\sigma_{\chi}$, as the result, the magnetic field will keep growing and finally blow up at some time. This of course breaks conservation laws. One way to avoid such divergences is to implement conservation laws in the system. This is the topic of the next subsection.

\section{B. With $g_{+}$only and dynamical $\sigma_{\chi}$}

We now consider imposing the total helicity conservation in Eq. (4). This has been implemented in Ref. [30, 37]. Here we focus on the approach to the CKW state in evolution. For simplicity, we can parameterize $\sigma_{\chi}$ as

$$
\sigma_{\chi}(t)=k_{h}\left[H_{\text {total }}-H(t)\right],
$$

where $k_{h}$ and $H_{\text {total }}$ (total helicity) are constants. From Eq. (50), we see that the requirement $\sigma_{\chi}>0$

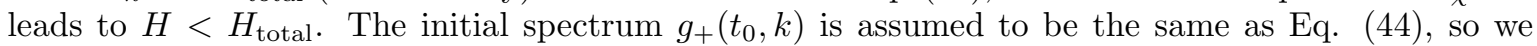
have $a(t)=\frac{\Theta(t)-L}{\sqrt{2 \Lambda(t)}}$. The parameters are chosen to be $\sigma=k_{h}=1 / L, t_{0}=0.1 L$, and $H_{0} / H_{\text {total }}=3 / 5$, where $H_{0}$ is given by Eq. (45). Since $\sigma$ is a constant, we have $\Lambda(t)=\frac{1}{\sigma}\left(t-t_{0}\right)$. We can solve $\sigma_{\chi}(t)$ self-consistently through $\Theta(t)$,

$$
\frac{d}{d t} \Theta(t)=\frac{k_{h}}{\sigma}\left\{H_{\text {total }}-H[\Theta(t)]\right\},
$$


where we have used $\sigma_{\chi}(t)=\sigma \frac{d}{d t} \Theta(t)$ and that $H$ depends on $\Theta(t)$ through $a(t)$ in Eq. (49).

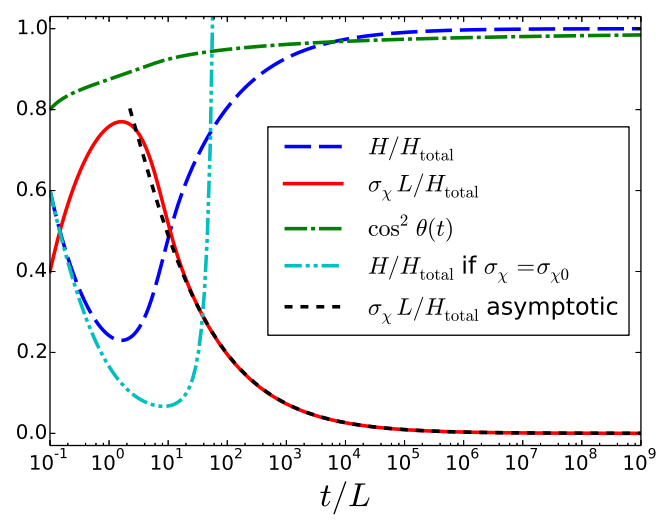

FIG. 3: (color online) The magnetic helicity $H$ (blue dashed), chiral magnetic conductivity $\sigma_{\chi}$ (red solid) and $\cos ^{2} \theta(t)$ (green dot-dashed) under the constraint $\sigma_{\chi} L+H=H_{\text {total }}$ as functions of $t$. The curve of $H$ (cyan dot-dot-dashed) is the result of constant chiral magnetic conductivity $\sigma_{\chi}=\sigma_{\chi 0}$. The black short dashed curve is the result by Eq. (54). As $\cos ^{2} \theta(t)$ approaches the limit $1, H$ takes all share of $H_{\text {total }}$, and $\sigma_{\chi}$ tends to decrease as Eq. (54) asymptotically.

The numerical results for $\sigma_{\chi}(t), H(t)$ and $\cos ^{2} \theta(t)$ are presented in Fig. 3. For comparison, we also show the result for constant $\sigma_{\chi}$ with $\sigma_{\chi}=\sigma_{\chi 0}=2 H_{\text {total }} /(5 L)$. In both cases, at the beginning, $a(t)=\frac{\Theta(t)-L}{\sqrt{2 \Lambda(t)}}$ is not large enough to make $G_{2}(a)$ grows faster than $\Lambda(t)^{3 / 2}$, which makes $H \sim G_{2} / \Lambda^{3 / 2}$ in Eq. (49) decrease with time. After $a$ grows large enough as time goes on, $H$ starts to increase after reaching a minimum. In the case of dynamical $\sigma_{\chi}$, according to Eq. (50), $H$ and $\sigma_{\chi}$ are complementary to each other to make up a seesaw system. In this system, the decreasing of $H$ at the beginning raises the value of $\sigma_{\chi}$ and makes $\Theta(t)$ and $a(t)$ grow faster. As the result, the turning point comes earlier than the case of constant $\sigma_{\chi}$. As $H$ keeps growing, $\sigma_{\chi}$ drops down leading to slower increase of $\Theta(t)$, which makes $H$ grows slower. At the end, $H$ is saturated to $H_{\text {total }}$ instead of blowing up.

Let us look at the asymptotic time behavior of $\sigma_{\chi}$ as $a \rightarrow \infty$. As the magnetic helicity $H$ is saturated to $H_{\text {total }}$ following Eq. (49), with $G_{2}(a) \sim \sqrt{\pi} a^{2} \exp \left(a^{2}\right)$ and $\Lambda(t) \sim \frac{1}{\sigma_{0}} t$ at late time, we obtain

$$
a^{2} \approx \operatorname{Plog}\left(c t^{3 / 2}\right)
$$

where $c=\frac{H_{\text {total }}}{\sqrt{2 \pi} H_{0} L^{3} \sigma_{0}^{3 / 2}}$ and Plog is called product logarithm, which is the inverse function of $f(x)=$ $x e^{x}$. From $a=\frac{\Theta(t)-L}{\sqrt{2 \Lambda(t)}}$, we obtain at very large $t$,

$$
\Theta(t) \approx \sqrt{\frac{2}{\sigma_{0}} t \mathrm{P} \log \left(c t^{3 / 2}\right)}
$$

Since the Plog term increases with $t, \Theta(t)$ is always growing faster than $\sqrt{\Lambda(t)} \sim \sqrt{t}$. Thus the conditions are satisfied and the CKW state can be reached.

Taking a derivative of $\Theta(t)$ with respect to $t$, we obtain $\sigma_{\chi}(t)$ at late time from Eq. (53),

$$
\sigma_{\chi}(t) \approx \sqrt{\frac{\sigma_{0}}{8 t} \mathrm{P} \log \left(c t^{3 / 2}\right)} \frac{5+2 \mathrm{P} \log \left(c t^{3 / 2}\right)}{1+\mathrm{P} \log \left(c t^{3 / 2}\right)} .
$$

The asymptotic behavior of $\sigma_{\chi}(t)$ is $\sigma_{\chi}(t) \approx \sqrt{\frac{\sigma_{0}}{2 t} \mathrm{Plog}\left(c t^{3 / 2}\right)}$ which decays slower than $t^{-1 / 2}$. We show the result from Eq. (54) in the black short dashed line in Fig. 3, which agrees with the numerical result very well.

We have also looked at a general spectrum for $g_{+}$at initial time,

$$
g_{+}\left(t_{0}, k\right)=N_{0} k^{r} e^{-2 k C_{1}-k^{2} C_{2}^{2}},
$$


where the normalization constant $N_{0}$ is determined by the initial magnetic helicity $H_{0}$. We assume $\sigma(t) \sim t^{-\alpha}$ obeying the power law decay in time, which gives $\Lambda(t) \sim t^{1+\alpha}$. In this case, solving Eq. (51) gives the late time asymptotic behavior,

$$
\Theta(t) \approx \sqrt{(r+1) \Lambda \mathrm{P} \log \left[\frac{4}{r+1}\left(\frac{\sqrt{2} H_{\text {total }}}{\sqrt{\pi} N_{0}}\right)^{2 /(r+1)} \Lambda^{(r+2) /(r+1)}\right]} .
$$

Again we see that $\Theta(t)$ grows faster than $\sqrt{\Lambda(t)}$ and the CKW state can be finally reached.

\section{With mixed helicity}

In this example we consider both positive and negative modes. We will show that only the positive mode survives while the negative mode decays away in late time. Let us consider the most extreme case in which the initial spectra of the positive and negative modes are the same. We take the following initial spectra for $g_{ \pm}$,

$$
g_{ \pm}\left(t_{0}, k\right)=4 H_{0} L^{3} k e^{-2 k L}
$$

It is obvious that the initial magnetic helicity is zero. Since $g_{ \pm}\left(t_{0}, k\right) \sim k, \cos ^{2} \theta(t)$ is given by Eq. (38) with $r=1$ and $a^{ \pm}(t)=\frac{ \pm \Theta(t)-L}{\sqrt{2 \Lambda(t)}}$. Obviously at the initial time we have $\cos ^{2} \theta\left(t_{0}\right)=0$ because $a^{+}\left(t_{0}\right)=a^{-}\left(t_{0}\right)$.
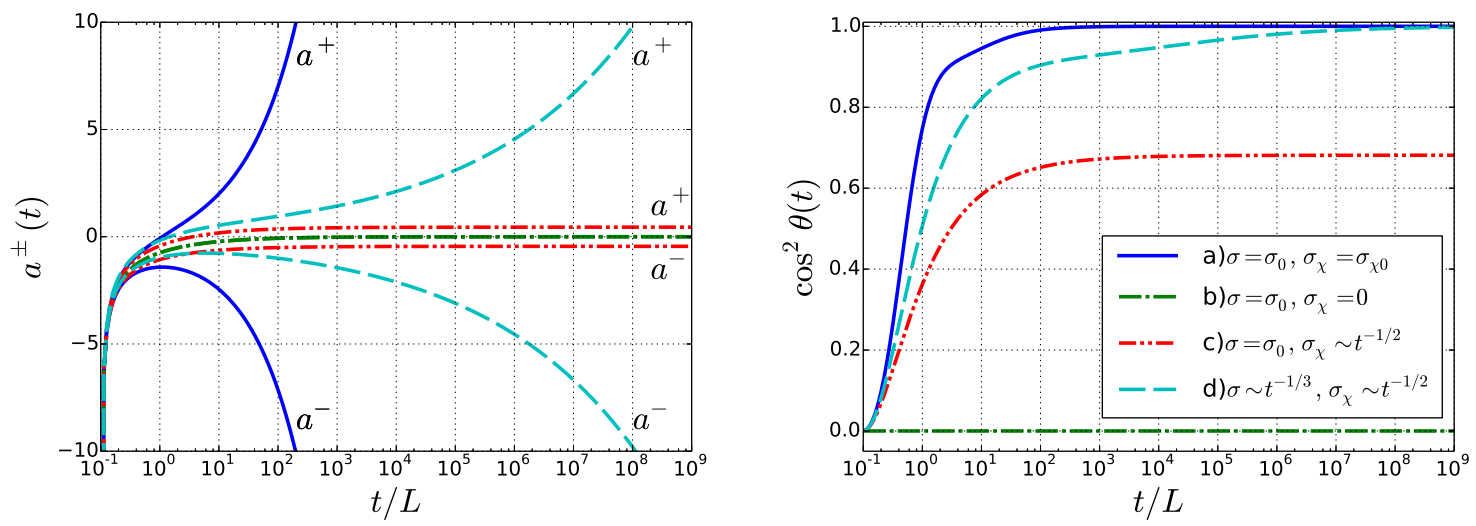

FIG. 4: (color online) The time behaviors of $a^{ \pm}(t)$ (left panel) and $\cos ^{2} \theta(t)$ (right panel) in different cases. In case a) (blue solid) and d) (cyan dashed), $\cos ^{2} \theta(t)$ tend to 1 in late time. In case c) (red dot-dot-dashed) $\cos ^{2} \theta(t)$ tends to a limit less than 1 , while in case b) (green dot-dashed) $\cos ^{2} \theta(t)$ is equal to 0 due to $a^{+}=a^{-}$.

We consider again the cases a)-d) as in (48). The results are shown in Fig. 4. The CKW state can be approached in case a) and d). As is shown in the left panel of Fig. 4, $a^{ \pm}(t)$ grow to $\pm \infty$ respectively, therefore at very late time the negative mode becomes highly suppressed and the positive mode survives, leading $\cos ^{2} \theta(t)$ to approach 1 . However in case b) we have $a^{+}=a^{-}$and $a^{ \pm} \rightarrow 0$ in late time. In this case the negative/positive modes are identical in the time evolution, which results in $\cos ^{2} \theta(t)=0$. In case $\mathrm{c}$ ) we have $\beta=\frac{1+\alpha}{2}$ which breaks the conditions for the CKW state. In this case, $a^{ \pm}(t)$ do not go to $\pm \infty$ at $t \rightarrow+\infty$, instead they approach the limits $\pm \frac{\sigma_{\chi 0}}{\sigma_{0}} \sqrt{2 t_{0} \sigma_{0}}$. We see that $\cos ^{2} \theta(t)$ keeps growing with time till it gets saturation, $\lim _{t \rightarrow \infty} \cos ^{2} \theta(t) \rightarrow 0.681$. All these results are consistent with our expectations. 


\section{MORE GENERAL MOMENTUM SPECTRA}

The power pre-factor $k^{r}$ of initial momentum spectra $g_{ \pm}\left(t_{0}, k\right)$ in (33) can be generalized to a polynomial,

$$
g_{ \pm}\left(t_{0}, k\right)=\left(\sum_{r=r_{\min }}^{r_{\max }} c_{r} k^{r}\right) e^{-2 k C_{1}} e^{-k^{2} C_{2}^{2}}
$$

where $\sum_{r} c_{r} k^{r}>0$ for any $k$ due to the fact that $g_{ \pm}$must be positive. Here $r$ are real numbers with $r>-3$. Ignoring negative modes, we obtain $\cos ^{2} \theta(t)$ from Eq. (39),

$$
\cos ^{2} \theta(t)=\frac{\left[\sum_{r=r_{\min }}^{r_{\max }} c_{r} G_{r+3}(a)\right]^{2}}{\left[\sum_{r=r_{\min }}^{r_{\max }} c_{r} G_{r+4}(a)\right] \times\left[\sum_{r=r_{\min }}^{r_{\max }} c_{r} G_{r+2}(a)\right]},
$$

where $a \equiv a^{+}$. According to Eq. (A6), the $r_{\min }$-th term is dominant at $a \rightarrow-\infty$, while the $r_{\max }$-th term is dominant at $a \rightarrow+\infty$. We can prove that $\cos ^{2} \theta(t)$ in Eq. (59) tends toward $\left(r_{\min }+3\right) /\left(r_{\min }+4\right)$ at $a \rightarrow-\infty$ by Eq. A77. From Eq. (A9), at large positive $a$ the ratio $\left(G_{n}\right)^{2} /\left(G_{n+1} G_{n-1}\right)$ can be approximated by $1-1 /\left(2 a^{2}\right)$. Hence the right-hand-side of Eq. (59) dominated by $\left(G_{r_{\max }+3}\right)^{2} /\left(G_{r_{\max }+4} G_{r_{\max }+2}\right)$ is also approximately $1-1 /\left(2 a^{2}\right)$ at large positive $a$ and reaches 1 for $a \rightarrow+\infty$. So our conditions for the CKW state are applicable to the initial momentum spectrum (58) with a polynomial pre-factor.

As examples we consider following initial momentum spectra $g_{+}\left(t_{0}, k\right) \sim k+k^{2}, k+k^{10}$ and $k-2 k^{2}+k^{3}$. We compare them with the power pre-factor $g_{+}\left(t_{0}, k\right) \sim k$ we considered in the last section. We use Eq. (59) for $\cos ^{2} \theta(t)$. In all these cases we have $r_{\min }=1$ and $\left(r_{\min }+3\right) /\left(r_{\min }+4\right)=0.8$.

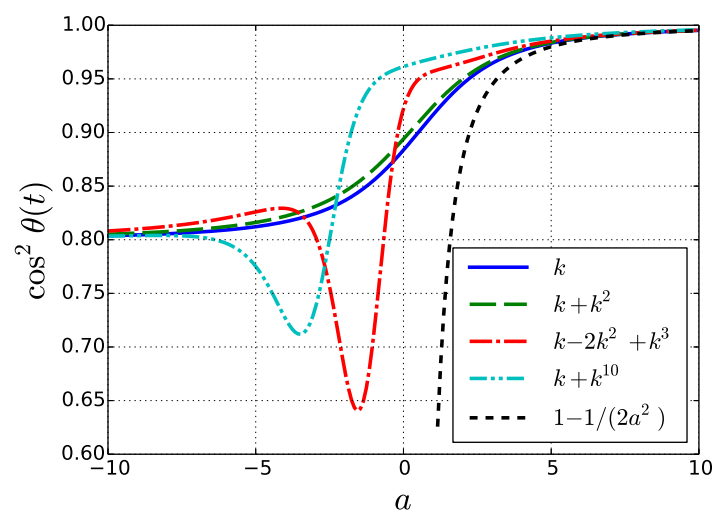

FIG. 5: (color online) The quantity $\cos ^{2} \theta(t)$ in Eq. (59) as functions of $a$ for $g_{+}\left(t_{0}, k\right) \sim k, k+k^{2}, k-2 k^{2}+k^{3}$ and $k+k^{10}$. They are all near 0.8 at $a \rightarrow-\infty$ and fit $1-1 /(2 a)^{2}$ at large positive $a$.

We plot $\cos ^{2} \theta(t)$ as functions of $a$ in Fig. 5. The results show that at $a \rightarrow-\infty$, all curves are near 0.8 which implies the $r_{\min }$-th term is dominant. For moderate values of $a$, the curves for different initial momentum spectra diverge significantly. At large positive $a$, they converge again and approach $1-1 /(2 a)^{2}$ as expected.

\section{SUMMARY AND CONCLUSIONS}

We investigate the Chandrasekhar-Kendall-Woltjer (CKW) state in a plasma with currents induced by chiral anomaly in magnetic fields. We solve the Maxwell-Chern-Simons equations and propose the conditions at which the CKW state can be finally realized in time evolution. We decompose the vector potential and the magnetic field into vector spherical harmonic modes. These modes are labeled by scalar momentum $k=|\mathbf{k}|$, quantum numbers of orbital angluar momentum $l$ and angular momentum along specific direction $m$, and the photon (electromagnetic field) helicity $s= \pm$. For each mode, there is an ordinary differential equation in time whose solution can be easily found. 
We define a quantity $\cos ^{2} \theta(t)$ for the CKW state, where $\theta(t)$ can be regarded as the average angle between $\mathbf{j}$ and $\mathbf{B}$. If $\cos ^{2} \theta(t)=1$, the CKW state is reached. In the real world the CKW state cannot be exactly reached but only be approached asymptotically, i.e. $\lim _{t \rightarrow+\infty} \cos ^{2} \theta(t)=1$. In the bases of vector spherical harmonic functions, a general inner product of two vector fields can be put into a uniform integral, from which we can express $\cos ^{2} \theta(t)$ in a simple form.

We propose the conditions that the CKW state can be reached in time evolution for a general class of initial momentum spectra: (i) the presence of $\sigma_{\chi}$ and (ii) $\Theta(t)$ grows faster in time than $\sqrt{\Lambda(t)}$, where $\Lambda(t)$ and $\Theta(t)$ are integrations over $t$ of $1 / \sigma$ and of $\sigma_{\chi} / \sigma$ respectively.

We take some examples to test these conditions. The numerical results agree with these conditions very well. In numerical calculations, we set different values for the parameters $\sigma_{0}, \sigma_{\chi 0}, t_{0}, k_{h}$, $H_{0} / H_{\text {total }}$ and the powers of $k$ in initial momentum spectra. We find that in cases of constant $\sigma_{\chi}$ and some power law decaying functions, although the CKW state can be approached but the magnetic helicity and magnetic energy will blow up. Such a problem can be avoided with dynamical $\sigma_{\chi}$ determined self-consistently with a helicity bound and a negative feedback. The critical decaying behavior of $\sigma_{\chi}$ given in Eq. (54) is found both numerically and analytically. The CKW state can be reached at the end of time evolution.

Acknowledgments. QW and XLX thank H. Li and Y.G. Yang for helpful discussions. QW is supported in part by the Major State Basic Research Development Program (MSBRD) in China under Grant 2015CB856902 and 2014CB845402 respectively and by the National Natural Science Foundation of China (NSFC) under the Grant 11535012.

\section{Appendix A: Properties of function $G_{n}(a)$}

We give some useful properties of the function $G_{r+n}\left(a^{ \pm}\right)$in Eq. (36). For convenience we simply denote this function as $G_{n}(a)$ in this appendix. We formally define $G_{n}(a)$ as

$$
G_{n}(a)=\int_{0}^{\infty} d \kappa \kappa^{n} e^{-\kappa^{2}+2 a \kappa}
$$

where $n$ is any real number larger than -1 .

By definition $G_{n}(a)$ can be integrated as

$$
G_{n}(a)=a \Gamma\left(\frac{n+3}{2}\right){ }_{1} F_{1}\left(\frac{n+3}{2} ; \frac{3}{2} ; a^{2}\right)+\frac{1}{2} \Gamma\left(\frac{n+1}{2}\right){ }_{1} F_{1}\left(\frac{n+1}{2} ; \frac{1}{2} ; a^{2}\right),
$$

where $\Gamma(x)$ is the gamma function and ${ }_{1} F_{1}(a ; b ; x)$ is the confluent hypergeometric function of the first kind. The above formula is applicable for any real number $n>-1$. For $n$ being integer, $G_{n}(a)$ can also be determined recursively by

$$
G_{n}(a)=a G_{n-1}(a)+\frac{n-1}{2} G_{n-2}(a),
$$

with

$$
\begin{aligned}
& G_{0}(a)=\frac{\sqrt{\pi}}{2} \exp \left(a^{2}\right) \operatorname{erfc}(-a), \\
& G_{1}(a)=\frac{1}{2}\left[1+\sqrt{\pi} a \exp \left(a^{2}\right) \operatorname{erfc}(-a)\right],
\end{aligned}
$$

where $\operatorname{erfc}(x)$ is the complementary error function, $\operatorname{erfc}(x)=\frac{2}{\sqrt{\pi}} \int_{x}^{\infty} d t \exp \left(-t^{2}\right)$.

From the definition Eq. (A1), it is straightforward to have

$$
\frac{d}{d a} G_{n}(a)=2 G_{n+1}(a)
$$

Since $G_{n+1}(a)$ are always positive, $G_{n}(a)$ are monotonically increasing functions of $a$. We plot $G_{n}(a)$ in Fig. 6 for $n$ being integers $0,1,2$ and a real number 3.5 as examples. They all approach zero at $a \rightarrow-\infty$, but rise sharply to $+\infty$ at $a \rightarrow+\infty$.

For the purpose of this paper, we are concerned with the asymptotic behavior of $G_{n}(a)$ at $a \rightarrow \infty$. From Eq. (A2) we have found that

$$
\lim _{a \rightarrow+\infty} \frac{G_{n}(a)}{\sqrt{\pi} a^{n} \exp \left(a^{2}\right)}=\lim _{a \rightarrow-\infty} \frac{(-2 a)^{n+1}}{\Gamma(n+1)} G_{n}(a)=1 .
$$



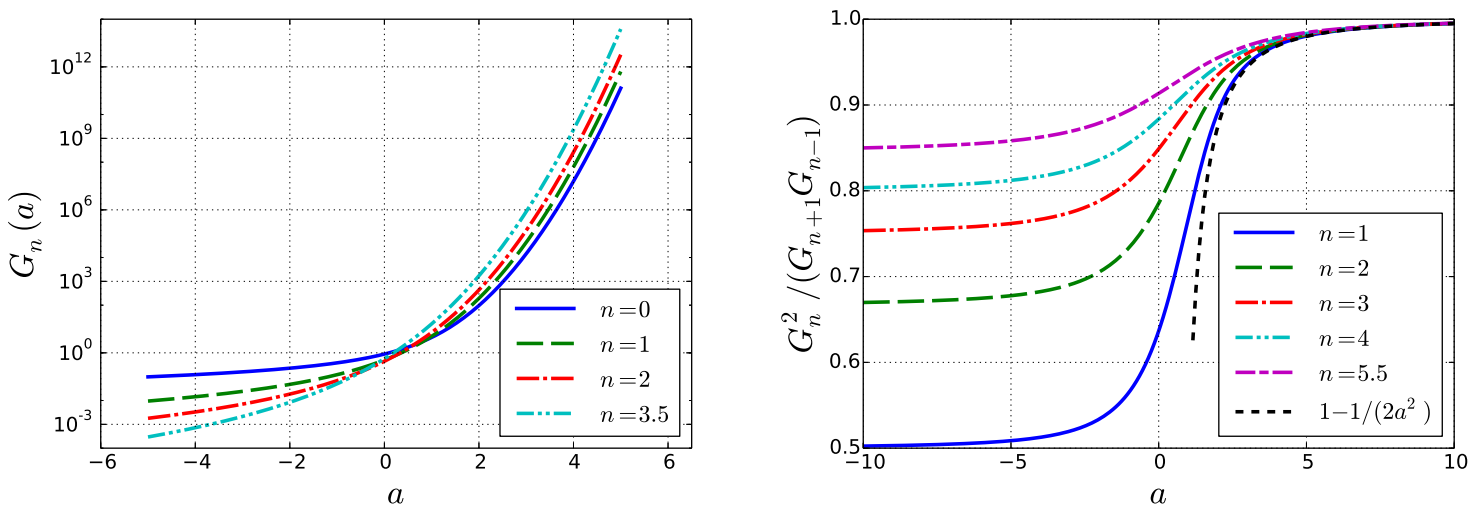

FIG. 6: (color online) Left panel: $G_{n}(a)$ as functions of $a$ for $n=0,1,2,3.5$. They tend to 0 at $a \rightarrow-\infty$ and $+\infty$ at $a \rightarrow+\infty$. Right panel: $\left[G_{n}(a)\right]^{2} /\left[G_{n-1}(a) G_{n+1}(a)\right]$ as functions of $a$. They increase from $\frac{n}{n+1}$ to 1 in the range $a \in(-\infty,+\infty)$ and fit asymptote $1-1 /(2 a)^{2}$ for large positive $a$.

This means $G_{n}(a)$ approach $\sqrt{\pi} a^{n} \exp \left(a^{2}\right)$ and $\Gamma(n+1)\left(-\frac{1}{2 a}\right)^{n+1}$ at $a \rightarrow \pm \infty$ respectively, and implies that

$$
\lim _{a \rightarrow+\infty} \frac{\left[G_{n}(a)\right]^{2}}{G_{n+1}(a) G_{n-1}(a)}=1, \quad \lim _{a \rightarrow-\infty} \frac{\left[G_{n}(a)\right]^{2}}{G_{n+1}(a) G_{n-1}(a)}=\frac{n}{n+1} .
$$

We plot the quantity $\left[G_{n}(a)\right]^{2} /\left[G_{n+1}(a) G_{n-1}(a)\right]$ in Fig. [6 for $n=1,2,3,4,5.5$. It shows that they increase with $a$ in S-shape from $\frac{n}{n+1}$ to 1 in the range $a \in(-\infty,+\infty)$.

To obtain the asymptote of $\left[G_{n}(a)\right]^{2} /\left[G_{n+1}(a) G_{n-1}(a)\right]$ at $a \rightarrow+\infty$, we expand $G_{n}(a)$ for large $a$ to the next to leading order in $1 / a$,

$$
G_{n}(a) \approx \sqrt{\pi} a^{n}\left[1+\frac{n(n-1)}{4 a^{2}}\right] \exp \left(a^{2}\right)
$$

So the asymptote of the the ratio $\left[G_{n}(a)\right]^{2} /\left[G_{n+1}(a) G_{n-1}(a)\right]$ is

$$
\begin{aligned}
\frac{\left[G_{n}(a)\right]^{2}}{G_{n+1}(a) G_{n-1}(a)} & \approx \frac{\left[1+n(n-1) /\left(4 a^{2}\right)\right]^{2}}{\left[1+n(n+1) /\left(4 a^{2}\right)\right]\left[1+(n-1)(n-2) /\left(4 a^{2}\right)\right]} \\
& \approx \frac{1+\left(n^{2}-n\right) /\left(2 a^{2}\right)}{1+\left(n^{2}-n+1\right) /\left(2 a^{2}\right)} \\
& =1-\frac{1}{2 a^{2}+n^{2}-n+1} \\
& \approx 1-\frac{1}{2 a^{2}} .
\end{aligned}
$$

Fig. [6] shows that all the quantity $\left[G_{n}(a)\right]^{2} /\left[G_{n+1}(a) G_{n-1}(a)\right]$ approach the asymptote $1-1 /(2 a)^{2}$ for large $a$. We see with the larger $n^{2}-n+1$, they deviate the asymptote further. At very large $a$, they fit $1-1 /(2 a)^{2}$ very well. We also see $\left[G_{n}(a)\right]^{2} /\left[G_{n+1}(a) G_{n-1}(a)\right]$ tend toward 1 if and only if $a \rightarrow+\infty$

[1] D. E. Kharzeev, L. D. McLerran, and H. J. Warringa, Nucl. Phys. A803, 227 (2008), 0711.0950.

[2] V. Skokov, A. Yu. Illarionov, and V. Toneev, Int. J. Mod. Phys. A24, 5925 (2009), 0907.1396.

[3] V. Voronyuk, V. D. Toneev, W. Cassing, E. L. Bratkovskaya, V. P. Konchakovski, and S. A. Voloshin, Phys. Rev. C83, 054911 (2011), 1103.4239.

[4] W.-T. Deng and X.-G. Huang, Phys. Rev. C85, 044907 (2012), 1201.5108.

[5] J. Bloczynski, X.-G. Huang, X. Zhang, and J. Liao, Phys. Lett. B718, 1529 (2013), 1209.6594. 
[6] L. McLerran and V. Skokov, Nucl. Phys. A929, 184 (2014), 1305.0774.

[7] U. Gursoy, D. Kharzeev, and K. Rajagopal, Phys. Rev. C89, 054905 (2014), 1401.3805.

[8] V. Roy and S. Pu, Phys. Rev. C92, 064902 (2015), 1508.03761.

[9] K. Tuchin, Phys. Rev. C91, 064902 (2015), 1411.1363.

[10] H. Li, X.-l. Sheng, and Q. Wang (2016), 1602.02223.

[11] K. Fukushima, D. E. Kharzeev, and H. J. Warringa, Phys. Rev. D78, 074033 (2008), 0808.3382.

[12] D. E. Kharzeev, J. Liao, S. A. Voloshin, and G. Wang, Prog. Part. Nucl. Phys. 88, 1 (2016), 1511.04050.

[13] B. I. Abelev et al. (STAR), Phys. Rev. Lett. 103, 251601 (2009), 0909.1739.

[14] B. I. Abelev et al. (STAR), Phys. Rev. C81, 054908 (2010), 0909.1717.

15] B. Abelev et al. (ALICE), Phys. Rev. Lett. 110, 012301 (2013), 1207.0900.

[16] X.-G. Huang, Y. Yin, and J. Liao, in 25th International Conference on Ultra-Relativistic Nucleus-Nucleus Collisions (Quark Matter 2015) Kobe, Japan, September 27-October 3, 2015 (2015), 1512.06602, URL https://inspirehep.net/record/1410880/files/arXiv:1512.06602.pdf.

[17] D. T. Son and B. Z. Spivak, Phys. Rev. B88, 104412 (2013), 1206.1627.

[18] G. Basar, D. E. Kharzeev, and H.-U. Yee, Phys. Rev. B89, 035142 (2014), 1305.6338

[19] Q. Li, D. E. Kharzeev, C. Zhang, Y. Huang, I. Pletikosic, A. V. Fedorov, R. D. Zhong, J. A. Schneeloch, G. D. Gu, and T. Valla, Nature Phys. 12, 550 (2016), 1412.6543.

[20] S. Chandrasekhar, Proceedings of the National Academy of Sciences 42, 1 (1956).

[21] S. Chandrasekhar and P. Kendall, The Astrophysical Journal 126, 457 (1957).

[22] S. Chandrasekhar and L. Woltjer, Proceedings of the National Academy of Sciences 44, 285 (1958).

[23] L. Woltjer, Proceedings of the National Academy of Sciences 44, 489 (1958).

[24] M. N. Chernodub (2010), 1002.1473.

[25] J. B. Taylor, Phys. Rev. Lett. 33, 1139 (1974).

[26] J. B. Taylor, Reviews of Modern Physics 58, 741 (1986).

[27] S. Ortolani and D. D. Schnack, Magnetohydrodynamics of Plasma Relaxation (World Scientific, Singapore, 1993), p. 56.

[28] H. Qin, W. Liu, H. Li, and J. Squire, Phy. Rev. Lett. 109, 235001 (2012).

[29] J. D. Jackson, Classical electrodynamics (Wiley, 1999).

[30] Y. Hirono, D. Kharzeev, and Y. Yin, Phys. Rev. D92, 125031 (2015), 1509.07790.

[31] K. Tuchin, Phys. Rev. C93, 054903 (2016), 1601.05399.

[32] W. T. Irvine and D. Bouwmeester, Nature Physics 4, 716 (2008).

[33] K. Tuchin, Adv. High Energy Phys. 2013, 490495 (2013), 1301.0099.

[34] H. T. Ding, A. Francis, O. Kaczmarek, F. Karsch, E. Laermann, and W. Soeldner, Phys. Rev. D83, 034504 (2011), 1012.4963.

[35] D. E. Kharzeev and H. J. Warringa, Phys. Rev. D80, 034028 (2009), 0907.5007.

[36] N. Yamamoto, Phys. Rev. D93, 125016 (2016), 1603.08864.

[37] C. Manuel and J. M. Torres-Rincon, Phys. Rev. D92, 074018 (2015), 1501.07608.

[38] Y. Akamatsu and N. Yamamoto, Phys. Rev. Lett. 111, 052002 (2013), 1302.2125. 